\title{
THE ECONOMICS OF SMALL WORLDS
}

\author{
Matthew O. Jackson \\ California Institute of Technology
}

\author{
Brian W. Rogers \\ California Institute of Technology
}

\begin{abstract}
We examine a simple economic model of network formation where agents benefit from indirect relationships. We show that small-world features - short path lengths between nodes together with highly clustered link structures-necessarily emerge for a wide set of parameters. (JEL: D85, A14, C72)
\end{abstract}

\section{Introduction}

Network structures play a central role in determining outcomes in many important situations. Examples include the Internet, coauthor relationships among academics, joint research venture projects among firms, trade networks, the sharing of job opening (and other sorts of) information through social networks, and P2P systems for file sharing. Given the large and increasing prevalence of situations where network structure are important, it is necessary to understand the properties of these networks and how various aspects of the network formation process determine those properties.

A variety of large social networks have been shown to exhibit certain characteristics. In this paper we explore two prominent ones that together embody what has been coined as "small worlds" (see Milgram 1967 and Watts 1999). The first characteristic is that such networks have small diameter and small average path lengths. ${ }^{1}$ The second characteristic is that such networks have high

Acknowledgments: Financial support under NSF Grant no. SES-0316493 is gratefully acknowledged, as is support from the Lee Center for Advanced Networking and a SISL/IST fellowship. We thank Fernando Vega-Redondo for the opportunity to present in this session and, along with Matthias Dahm and an anonymous referee, for comments on an earlier version of this paper.

E-mail addresses: Jackson: jacksonm@hss.caltech.edu; Rogers: rogers@ @ss.caltech.edu

1. The diameter is the maximum distance between any two nodes of the network, where distance between nodes is defined as the number of links in the shortest path between them. This stylized fact is captured in the famous "six degrees of separation" of John Gaure's play. Stanley Milgram (1967) pioneered the study of path length through a clever experiment in which people had to send a letter to another person who was not directly known to them. 
clustering coefficients relative to networks generated by an independent random process. $^{2}$

A number of models have been built to explain these (and other) characteristics of social networks (see Jackson and Rogers 2004 for a discussion and list of references). One prominent study is by Watts and Strogatz (1998). They show that if one starts with a symmetric (regular) network where nodes are connected to their nearest neighbors and where clustering is high, it takes only a small amount of random rewiring of links in order to drastically reduce the network's diameter and thus obtain a small world. Klemm and Eguíluz (2002a, 2002b) find high clustering in a model where nodes enter over time and only connect to other nodes in a small set that are considered "active", where this active set evolves over time. High clustering and low diameter can also be explained via a model that incorporates random linking combined with links formed through local search along network paths, as shown by Jackson and Rogers (2004).

While these models can yield small-world characteristics, they are "mechanical" models, where a particular process of link formation (or reformation) is specified, but there is not much explanation about why networks might form in accordance with such processes. ${ }^{3}$ This paper examines economic-based reasoning for small worlds. We consider a model where links generate explicit costs and benefits for agents, and then determine what networks will form when agents form links in their self-interest. We analyze how the small-world features can be traced to particulars of how the costs and benefits to agents vary.

We do not view "economic" models as a substitute for the more mechanical models of network formation, but rather as a complement. The simple model presented here captures some general features that one might expect many network situations to exhibit. Yet the model is highly stylized, and the networks that are formed are much too regular to resemble "real" networks. So while the model provides some insights into the "why" behind small-world characteristics, it is far too special to be a definitive model of real social networks. In contrast, some of the mechanical models contain enough randomness and heterogeneity to produce networks that appear closer to real networks, but they provide less insight into why networks form as they do. By examining both types of models, we can better understand how network structure is influenced by the (mechanical) node-meeting process and by the (economic) incentives of agents to form links.

From the mechanical side it is known that in some situations a few random connections between distant nodes can dramatically decrease network diameter, while from the economic side we learn that in some situations, distant nodes

2. Clustering coefficients measure the frequency with which two neighbors of a given node are themselves connected. Ideas behind clustering have been important in sociology since Simmel (1908), who pointed out the interest in triads. For more discussion on clustering and some empirical examples, see Watts (1999).

3. The Jackson and Rogers (2004) model can be seen to be consistent with utility maximization.

$$
\text { “zwu0250” — 2005/5/21 — page } 618 \text { — \#2 }
$$


greatly benefit (in terms of net utility) from forming links precisely because of the distance, which provides an answer as to why such shortcuts might be formed. The fundamental intuitions that emerge from the economic side are: (i) high clustering results from low costs of attachment to similar (nearby) nodes, and (ii) low diameter results from the large benefit of attaching to dissimilar (distant) nodes because of the substantial indirect access they provide to other distant nodes. A limited number of such distant links emerge due to the high costs, but in concert with the high interconnection rate at the local level, these distant links substantially decrease networks diameter and average path length.

The model here is built on a variation of the connections model of Jackson and Wolinsky (1996), where agents benefit from their direct connections and also from indirect connections. That is, friends of a friend generate value and so on. The departure from that model is that we describe a simple geographically based cost structure to forming links. This cost structure captures heterogeneity in link costs in a simple manner: agents are grouped on "islands", and costs of connection are relatively low within an island and relatively high across islands. This cost structure, together with the indirect benefits structure of the connections model, generates the small-world characteristics.

There are other economic studies that have looked at the connections model with geographic costs, such as Johnson and Gilles (2000), Carayol and Roux (2003), and Galeotti, Goyal, and Kamphorst (2004). The first two papers examine situations where agents are arranged on a line or circle and link costs are proportional to distance. Intuitively, such models generate higher rates of connection on a local scale, and due to the positive indirect benefits from connection they sustain some distant connections, thus exhibiting small-world features. Indeed, Carayol and Roux (2003) show that, in certain situations, some of the pairwise stable networks exhibit small-world characteristics, and through simulation that, at least for one parameterization, stochastic stability tends to select small-world networks. Thus, the important insight - that connections-like models can result in small-world networks-is not new to our paper but rather is due to Carayol and Roux (2003). ${ }^{4}$ The model of Galeotti, Goyal and Kamphorst (2004) allows for a more general geographic cost structure than those cited above, and includes as a special case the cost structure that we analyze here. However, Galeotti, Goyal, and Kamphorst focus on the case where benefits decay negligibly with distance, so that any equilibrium network is minimally connected. Because minimally connected networks exhibit no clustering, their results are in contrast to what we find here, where decay is a central feature of the model and clustering is an important aspect of the emerging networks.

4. Such characteristics can also be seen in some of the networks found to be pairwise stable by Johnson and Gilles (2000), but this aspect was not their focus.

$$
\text { "zwu0250" — 2005/5/21 — page } 619 \text { — \#3 }
$$


The simple islands-based variation of the (truncated) connections model is much more tractable than other geographic variations of the connections model. This allows us to obtain a more complete picture of the networks that emerge in equilibrium and to show that all stable and, in fact, all efficient networks necessarily exhibit the small-world characteristics of small diameter and high clustering.

\section{Background Definitions}

\subsection{Networks}

Let $N=\{1,2, \ldots, n\}$ denote a set of agents, which we identify with nodes. A network $g$ is a list of unordered pairs of agents $\{i, j\}$ describing which agents are connected. For simplicity, write $i j$ to represent the link $\{i, j\}$, so $i j \in g$ indicates that $i$ and $j$ are linked under the network $\mathrm{g}$. A shorthand notation for the network obtained by adding (deleting) link $i j$ to an existing network $g$ is $g+i j(g-i j)$.

For any network $\mathrm{g}$ and agent $i$, let $N_{i}(\mathrm{~g})$ be the neighborhood of $i$ under $g$, that is, the set of agents linked to $i$ in the network $g$, so that $N_{i}(g)=\{j \mid i j \in g\}$.

\subsection{Utility and Efficiency}

Let $u_{i}(g)$ (defined below) denote the net utility that agent $i$ receives under the network $g$, inclusive of all costs and benefits.

A network is efficient if it maximizes $\sum_{i} u_{i}(g)$. This is a strong definition of efficiency, which corresponds to a utilitarian measure (or Pareto 1896 if transfers are unrestricted).

\subsection{Pairwise Stability}

A network $g$ is pairwise stable if

(i) for all $i j \in g, u_{i}(g) \geq u_{i}(g-i j)$ and $u_{j}(g) \geq u_{j}(g-i j)$, and

(ii) for all $i j \notin g$, if $u_{i}(g+i j)>u_{i}(g)$ then $u_{j}(g+i j)<u_{j}(g)$.

Pairwise stability captures the idea that mutual consent is necessary to form or maintain a link. A network is stable if no two individuals both want to add a link and no single individual wants to sever a link. This is a weak requirement; it does not, for instance, consider changes in multiple links at the same time. ${ }^{5}$

5. See Jackson (2004) for a review of some of the different methods that have been used to study strategic network formation.

$$
\text { "zwu0250" — 2005/5/21 — page } 620 \text { — \#4 }
$$


Nevertheless, since our results will show that all pairwise stable networks turn out to exhibit small-world features, the results are strengthened by the use of a stability concept that is permissive.

\subsection{Distance and Diameter}

A path in a network $g \in G$ between agents $i$ and $j$ is a sequence of agents $i_{1}, \ldots, i_{K}$ such that $i_{k} i_{k+1} \in g$ for each $k \in\{1, \ldots, K-1\}$ with $i_{1}=i$ and $i_{K}=j$. The length of such a path is $K-1$, the number of links involved. The distance between two agents $i$ and $j$ denoted $d(i, j)$, is the minimum path length between $i$ and $j$ (and set to be infinite if no such path exists). The diameter of a network $g$ is then defined as $\bar{d}(g)=\max _{i j \in N: d(i, j)<\infty} d(i, j)$, the maximum distance between any two connected nodes.

\subsection{Clustering}

There are a variety of ways that clustering has been measured. One is a wellknown measure from the sociology literature (see, e.g., Wasserman and Faust 1994) that examines the percentage of "transitive triples." This technique looks at cases where node $i$ has a link to $j$ and $j$ has a link a to $k$, and then asks whether $i$ has a link to $k$. The percentage of times in a network that the answer is "yes" is the fraction of transitive triples. ${ }^{6}$ To distinguish this from the other common notion of clustering, we call this total clustering, defined as

$$
C(g)=\frac{\sum_{i} \#\left\{j k \in g \mid k \neq j, j \in N_{i}(g), k \in N_{i}(g)\right\}}{\sum_{i} \#\left\{j k \mid k \neq j, j \in N_{i}(g), k \in N_{i}(g)\right\}} .
$$

Another measure, which is sometimes easier to compute on a given network, is a variation whereby clustering is measured in the neighborhood of each individual node and then averaged across individuals (see, e.g., Watts 1999). We refer to this as average clustering, which is based on the following definition of individual clustering for a node $i$ :

$$
C_{i}(g)=\frac{\#\left\{j k \in g \mid k \neq j, j \in N_{i}(g), k \in N_{i}(g)\right\}}{\#\left\{j k \mid k \neq j, j \in N_{i}(g), k \in N_{i}(g)\right\}} .
$$

The average clustering coefficient is then

$$
C^{a v g}(g)=\sum_{i} \frac{C_{i}(g)}{n} .
$$

6. This fraction has been referred to as "clustering" in the recent statistical physics literature (e.g., see the survey by Newman 2003 and is also sometimes referred to as "cliquishness."

$$
\text { "zwu0250" — 2005/5/21 — page 621 — \#5 }
$$




\section{Small Worlds in an Islands-Connections Model}

We examine a simple "islands" version of the (truncated) connections model of Jackson and Wolinsky (1996). There are $K$ islands, each with $J$ agents on it. Forming a link between agents $i$ and $j$ costs each of the agents $c$ if $i$ and $j$ are on the same island and $C$ otherwise, where $C>c>0$.

This geography provides a simple way of introducing heterogeneity among agents. Distance can be thought of as representing actual physical separation, but can also describe differences among agents in terms of social or political preferences, research interests, compatibility of $\mathrm{R} \& \mathrm{D}$ programs, and so forth.

There are many interpretations of the benefits, but one way to think of a link is as a social relationship that can offer benefits in terms of favors, information, and the like. The important aspect is that agents also benefit from indirect relationships: a friend of a friend relationship confers benefits, although of a lesser value than a direct friendship, as do "friend of a friend of a friend" relationships, and so forth. The benefit deteriorates geometrically with the distance of the relationship, as described by the parameter $\delta \leq 1$. However, agents pay costs only for maintaining their direct relationships.

The overall utility to an agent $i$ in network $g$ is

$$
u_{i}(g)=\sum_{j \neq i: d(i, j) \leq D} \delta^{d(i, j)}-\sum_{j: i j \in g} c_{i j}
$$

where $c_{i j}=c$ if $i$ and $j$ are on the same island and $c_{i j}=C$ otherwise. ot, $^{7}$

This version of the connections model truncates benefits at a length $D$. Thus, no indirect benefits are obtained from other agents who are at a distance of more than $D$. For large $D$, the basic connections model and the truncated connections model can be quite similar. The truncation here seems natural and is particularly helpful in making the results simple to present and prove. We assume $D \geq 2$, since otherwise agents benefit only from direct links and the network issues are degenerate. When $D \geq K J-1$ the maximal distance is never a constraint, so the model is identical to the basic connections model.

7. This cost structure is the same as that of the insiders-outsiders model of Galeotti, Goyal and Kamphorst (2004).

8. There are some straightforward ways in which this could be extended and remain fairly tractable. For instance, one could have an island structure within an island structure, so that there are three or more cost levels, indicating at what level individuals inhabit the same island. A second extension is to allow individuals to belong to several islands at once and have costs determined by whether or not two agents have an island in common.

$$
\text { “zwu0250” — 2005/5/21 — page 622 — \#6 }
$$




\subsection{Small-World Characteristics of the Islands Connections Model}

We now show how the islands-connections model generates small diameter and high clustering, resulting in small-world networks.

PROPOSITION 1. If $c<\delta-\delta^{2}$ and $C<\delta+(J-1) \delta^{2}$, then any network that is pairwise stable and/or efficient is such that

1. the agents on any given island are completely connected to each other,

2. the diameter is no greater than $D+1$, and

3. a lower bound on individual, average, and total clustering is $\frac{(J-1)(J-2)}{J^{2} K^{2}} \cdot{ }^{9}$

Proof. If two agents on the same island are not connected in some network, then they would each gain at least $\delta-\delta^{2}-c>0$ by adding the link, and so the network cannot be pairwise stable or efficient. Now suppose that there are two agents (on distinct islands), say $i$ and $j$, such that $d(i, j) \geq D+2$. As just argued, in any pairwise stable or efficient network, $j$ is directly connected to all members of his island and so is $i$. Thus $i$ is at a distance of at least $D+1$ from each member of $j$ 's island and so enjoys no benefit from any of these agents; the same is true for $j$ from $i$ 's island. Thus, by linking to $j, i$ would gain at least $\delta+(J-1) \delta^{2}-C>0$ (and vice versa), so this cannot be pairwise stable or efficient.

We next show that a lower bound for an individual's clustering (and thus average clustering) occurs when each of an agent's inter-island links is involved in no transitive triples. To obtain the lower bound on clustering, we now note that in the case where at most one connection from a given agent to any other island exists, the worst case for the clustering calculation is when none of the node's distant links are themselves connected. All of the agent's pairs of intra-island neighbors are themselves neighbors, and none of the agent's pairs involving an inter-island neighbor are linked. Thus there are $(J-1)(J-2) / 2$ pairs of $i$ 's neighbors that are linked out of a maximal total of $(J+L-1)(J+L-2) / 2$ pairs of neighbors, if the individual has $L$ inter-island links. This leads to a lower bound of $(J-1)(J-2) /[(J+L-1)(J+L-2)]$. Noting that $L \leq J(K-1)$, we have a loose lower bound of $(J-1)(J-2) /(J+J(K-1))^{2}$, resulting in the claimed expression.

The lower bound on the total clustering coefficient is established as follows. For a given network, write $i$ 's clustering coefficient as $a_{i} / b_{i}$, where $a_{i}$ is the number of links among neighbors in $i$ 's neighborhood and $b_{i}$ is the number of pairs of neighbors in $i$ 's neighborhood. We have established a lower bound

9. For the bounds on clustering we assume that $\delta-\delta^{2} \neq C$. If $\delta-\delta^{2}=C$ then there is a great deal of indifference over links, and the set of pairwise stable networks explodes.

$$
\text { “zwu0250” — 2005/5/21 — page } 623 \text { - \#7 }
$$


for $a_{i} / b_{i}$. Note that total clustering is $\left.\left(\sum_{i} a_{i}\right) / \sum_{i} b_{i}\right)$ and that this is clearly greater than $\min _{i}\left(a_{i} / b_{i}\right){ }^{10}$

The case identified in Proposition 1 can be considered a small world in a rather strong sense. The diameter is bounded above by $D+1$, and average path lengths will be considerably smaller still since each island is fully connected. Next observe that average clustering is approximated by $K^{-2}$. Thus, clustering can remain large when $n$ is very large, provided that per-island population is not too small. In cases where $C$ is large enough so that the number of inter-island links is lower (bounded by $K J$ ), then the lower bound for clustering is even higher (on the order of $\left.(J /(J+K))^{2}\right)$; and then even for large $K$ relative to $J$, the clustering is much larger than one would observe in a completely random network (which goes to zero as the population grows, holding the probability of links constant, as first studied by Erdös and Rényi 1960).

We emphasize that Proposition 1 applies to networks that are either pairwise stable or efficient, and thus it shows that there are some similarities between these sets of networks. Obtaining the precise relationship between pairwise stable networks and efficient networks is more complex. We are able to characterize the efficient networks in some cases when the intra-island costs are low, and they show some interesting patterns. From this we can see that for some range of interisland costs the pairwise stable and efficient network coincide, whereas for other cost ranges the set of pairwise stable networks, though always exhibiting small-world features, can be quite varied.

Proposition 2. Let $c<\delta-\delta^{2}$. In any efficient network, each island is internally completely connected. Inter-island links in efficient networks are characterized as follows.

1. If $C<\delta-\delta^{2}$ then the unique efficient network is the completely connected network.

2. If $\delta-\delta^{2}<C<\delta-\delta^{3}$ and $K=2$, then the efficient networks are those such that there are exactly J links between the two islands, and on at least one island each agent is involved in exactly one of the J links.

3. If $\delta-\delta^{3}+2(J-L-1)\left(\delta^{2}-\delta^{3}\right)<C<\delta-\delta^{3}+2(J-L)\left(\delta^{2}-\delta^{3}\right)$ and $K=2$, then the efficient networks are those such that there are exactly $1 \leq L<J$ links between the two islands and no agent is involved in more than one of these links.

4. If $\delta-\delta^{3}+2(J-2)\left(\delta^{2}-\delta^{3}\right)<C$ and $K=2$, then the efficient networks have at most one link between the two islands.

10. It is straightforward to check that $\left(a_{1}+a_{2}\right) /\left(b_{1}+b_{2}\right) \geq \min \left(a_{1} / b_{1}, a_{2} / b_{2}\right)$. The result then follows by induction.

$$
\text { “zwu0250" — 2005/5/21 — page } 624 \text { — \#8 }
$$


Case 4 can be broken down further: for low costs there is exactly one link between the two islands; and for higher costs the islands are isolated. When there are many islands, the characterization of efficient networks gets a bit more complicated, as star like structures can be efficient. For instance, when $K$ is very large and $C<\delta-\delta^{3}$, completely connecting all agents within each island, and then connecting every agent on every island other than island 1 directly to the same agent on island 1 can be more efficient than having intra-island links that don't all pass through the same island.

Proof. Given that $c<\delta-\delta^{2}$, efficiency clearly requires all intra-island links to form. The only question concerns the configuration of inter-island links. If $C<\delta-\delta^{2}$, then clearly the unique efficient network is complete.

Now consider the case where $\delta-\delta^{2}<C<\delta-\delta^{3}$. Fix a pair of islands and call the number of links between them $L$. If $L<J$, then there is at least one agent on each island without a link to the other island. Connecting them adds at least $2\left(\delta-\delta^{3}-C\right)>0$. Thus, efficiency requires that $L \geq J$. If $L>J$ and not all agents on an island are involved in one of these links, then the links can be rearranged so that each agent on that island is involved in at least one link without reducing total utility. So suppose that all agents on one of the islands are involved in at least one link to the other island. There must be an agent on that island with more than one link to the other island. Removing one such link saves $2 C-2\left(\delta-\delta^{2}\right)>0$. So efficiency requires $L=J$ in this cost range. Consider the expression

$$
V\left(x_{1}, x_{2}, L\right)=2 L \delta+2\left[J x_{1}+J x_{2}-x_{1} x_{2}\right]\left(\delta^{2}-\delta^{3}\right)-2 L \delta^{2}+2 J^{2} \delta^{3},
$$

which is the utility obtained by the members of island 1 from connections to island 2 plus the reverse, where $x_{i} \leq L$ is the number of agents on island $i$ having links to the other island. Maximizing $V$ with respect to $x_{1}$ and $x_{2}$ when $L=J$ requires that $x_{1}=L$ or $x_{2}=L$, i.e., on at least one island all agents must have a link to the other island. Maximizing $V$ when $L<J$ requires that $x_{1}=x_{2}=L$, i.e., no agent can be involved in more than one link to the other island. Now, if there are $L$ links between two islands (where the links satisfy this condition), the utility that agents on one of the islands get from connections to agents on the other island is $(J-L)\left(L \delta^{2}+(J-L) \delta^{3}\right)+L\left(\delta+(J-1) \delta^{2}\right)$. The gain in utility from having $L+1$ links versus $L$ links is then $\delta-\delta^{3}+2(J-L-1)\left(\delta^{2}-\delta^{3}\right)$. The condition in part 3 then follows from having it be worthwhile to add $L$ links but not $L+1$.

If $\delta-\delta^{3}+2(J-2)\left(\delta^{2}-\delta^{3}\right)<C$, then any link beyond the first one between two islands will not be beneficial. Hence, in this case there is at most one link between any two islands, as stated in part 4 .

These results apply to cases where the intra-island cost of connections is low enough that agents are completely connected within their own island. The analysis

$$
\text { “zwu0250" - 2005/5/21 — page 625 — \#9 }
$$


becomes more complex when the intra-island connection cost rises, so that not all agents within an island are connected. Nevertheless, we can still establish some small-world characteristics for a higher cost range. For instance, we can deduce bounds on diameter as follows. Consider the case where $\delta-\delta^{2}<c<\delta$ and $C<\delta+(J-1) \delta^{D}$. Even though an individual island will not be completely connected, if two agents on the same island were at distance greater than $D$, then each would gain at least $\delta-c>0$ by connecting, so the network could not have been pairwise stable. In light of this, consider agents $i$ and $j$ on different islands and suppose that $d(i, j) \geq 2 D+1$. Then each would be at distance at least $D+1$ from every agent on the other's island and so would derive no benefit from them. Thus, $i$ and $j$ could each secure at least $\delta+(J-1) \delta^{D}-C>0$ by the adding a link to the other island. Therefore the diameter of all pairwise stable networks is no greater than $2 D$, and the average path length is smaller than this worst case distance.

While high intra-island costs do not conflict with obtaining small diameters, clustering is another matter. Positive clustering requires the presence of "triangles," where an agent's neighbors are connected to each other. Although this can occur in pairwise stable networks when $c>\delta-\delta^{2}$, it is not a general characteristic of pairwise stable networks in this cost range. ${ }^{11}$ We make two remarks in this regard. First, there is still a sense in which the resulting pairwise stable networks will be "clustered" despite the possible absence of triangles. Intuitively, clustering should measure the relative density of nearby links compared to far away links, but the common measures fail to capture this more general property. Our islands cost structure will still generate this kind of feature. Second, there is a sense in which "islands" should be defined by the (given) relative costs—so that agents placed in the same island are, in fact, precisely those who have low costs of linking to each other and thus will naturally tend to be clustered.

\section{Discussion}

In addition to the small-world properties there are other characteristics shared by many socially generated networks, such as a distribution of node degrees (number of links per node) that are approximately "scale-free" or follow a power law, at least in the upper tail. ${ }^{12}$ Thus, there tend to be many more nodes with very small and very large degrees than one would see if the links were formed independently. ${ }^{13}$

11. Note that this is not an artifact of the "islands" geography, since any connections-based model will have an analogous property involving the costs of the shortest links.

12. The degree of a node is the cardinality of its neighborhood. An example of a distribution satisfying a power law is the Pareto distribution, where the frequency of nodes with degree $d$ is proportional to $d^{-\gamma}$ for some $\gamma>1$. For and informative overview, see Mitzenmacher (2004).

13. See. Jackson and Rogers (2004) for a more complete list of the properties and references for them. 
In our model there is substantial room for variation in degree across nodesfor instance, in who forms inter-island links-as well as in the pattern of intraisland links. There is little in the models that explicitly ties down this structure. A conjecture is that a variation of the model that marries a random process to how nodes meet with the economic and strategic concerns analyzed here would begin to account for the degree distribution and could result in features consistent with observables.

\section{References}

Carayol, Nicolas and Pascale Roux (2003). "Collective Innovation' in a Model of Network Formation with Preferential Meeting." Working paper, Université Louis Pasteur and Université de Toulouse I.

Erdös, Paul and Alfréd Rényi (1960). "On the Evolution of Random Graphs.” Publication of the Mathmatical Institute of the Hungarian Academy of Sciences, 5, 17-61.

Galeotti, Andrea, Sanjeev Goyal, and Jurjen Kamphorst (2004). "Network Formation with Heterogeneous Players." Working paper, Tinbergen Institute.

Jackson, Matthew O. (2004). "A Survey of Models of Network Formation: Stability and Efficiency" In Group Formation in Economics; Networks, Clubs and Coalitions, edited by Galbrielle Demange and Myrna Wooders. Cambridge University Press. 〈http:// www.hss.caltech.edu/ jacksonm/netsurv.pdf $\rangle$.

Jackson, Matthew O. and Brian W. Rogers (2004). "Search and Random Attachment in the Formation of Large Networks." Working paper, Caltech, 〈http://www.hss.caltech. edu/ jacksonm/netpower.pdf $\rangle$.

Jackson, Matthew O. and Asher Wolinsky (1996). "A Strategic Model of Social And Economic Networks." Journal of Economic Theory, 71, 44-74.

Johnson, Cathleen and Robert P. Gilles (2000). "Spatial Social Network." Review of Economic Design, 5, 273-300.

Klemm, Konstantin and Victor M. Eguíluz (2002a). "Growing Scale-Free Networks with Small World Behavior." Physical Review E, 65, 036123.

Klemm, Konstantin and Victor M. Eguíluz (2002b). "Highly Clustered Scale-Free Networks." Physical Review E, 65, 057102.

Milgram, Stanley (1967). “The Small-World Problem.” Psychology Today, 2, 60-67.

Mitzenmacher, Michael (2004). "A Brief History of Generative Models for Power Law and Lognormal Distributions." Manuscript, 〈http://www.eecs.harvard.edu/ michaelm/ ListByYear.html .

Newman, Mark (2003). "The Structure and Function of Complex Networks." SIAM Review, $45,167-256$.

Pareto, Vilfredo (1896). Cours d'Economie Politique, Droz, Geneva.

Simmel, Georg (1908). Sociology: Investigations on the Forms of Sociation. Dunker \& Humblot, Berlin.

Wasserman, Stanley and Faust, Kathleen (1994). Social Network Analysis: Methods and Applications. Cambridge University Press.

Watts, Duncan (1999). Small Worlds. Princeton University Press.

Watts, Duncan and Stephen Strogatz (1998). "Collective Dynamics of 'Small-World' Networks." Nature, 393, 440-442. 